\title{
Characterizing Riverine Biofilms Using Scanning Transmission X-ray and Transmission Electron Microscopies
}

\author{
M. M. Reid, ${ }^{*}$ G. G. Leppard, ${ }^{* *}$ J. J. Dynes, ${ }^{* * *}$ G. D. W. Swerhone, ${ }^{* * * *}$ T. Tyliszczak, ${ }^{* * * * *}$ \\ T. Araki, ${ }^{* * * * *}$ D. R. Korber, ${ }^{* * * * * *}$ A. P. Hitchcock, ${ }^{* * *}$ and J. R. Lawrence**** \\ * Electron Microscopy Facility, Faculty of Health Sciences, McMaster University, Hamilton, ON, \\ L8N 3Z5, Canada. \\ ** National Water Research Institute, PO Box 5050, 867 Lakeshore Rd., Burlington, ON, L7R \\ 4A6, Canada. \\ *** Brockhouse Institute for Materials Research, McMaster University, Hamilton, ON, L8S 4M1, \\ Canada. \\ ****National Water Research Institute, 11 Innovation Blvd., Saskatoon, SK, S7N 3H5, Canada. \\ ***** Chemical Sciences Division, Lawrence Berkeley National Lab, Berkeley, CA, 94720 \\ ******Department of Applied Microbiology and Food Science, University of Saskatchewan, \\ Saskatoon, SK, Canada.
}

Biofilms, which can form on many moist surfaces, are a complex mixture of cells and extracellular polymeric substances (EPS). The EPS serve many functions, including: (i) sorption of organic (e.g., antimicrobial agents) and inorganic (e.g., metals) compounds; (ii) trapping of particulates such as organic debris, humic substances, clays and other minerals; (iii) compound transformations. $\mathrm{X}$-ray absorption spectroscopy using synchrotron light sources can identify and quantify chemical species in complex matrices at a spatial resolution of $50 \mathrm{~nm}$ [1]. The X-ray absorption spectral signals in scanning transmission X-ray microscopy (STXM) are used to map biomolecular structure and allow the correlation of elements such as $\mathrm{Ca}, \mathrm{K}, \mathrm{Fe}, \mathrm{Mn}, \mathrm{Ni}$, etc. with protein, lipid, carbohydrate and nucleic acid components of the biofilm [2]. Since the chemical and structural integrity of the sample after STXM analysis remains intact due to reduced radiation damage $[3,4]$, further analysis using transmission electron microscopy (TEM) is possible. Conventional TEM is useful for studying the microstructure of biofilms at high resolution and can provide analytical information on the elemental composition of a sample. These approaches provide a combination of suitable spatial resolution and chemical information at the microscale and may be used to create a detailed correlative map of biofilm structure and composition (Fig. 1). Our studies are intended to improve understanding of the organization of biofilms and their capacity to sequester trace organic and inorganic compounds from the surrounding environment.

\section{$\underline{\text { References }}$}

[1] A. P. Hitchcock et al., J. Electron Spectrosc. (2005) in press.

[2] J. R. Lawrence et al., Appl. Environ. Microbiol. 69 (2003) 5543.

[3] E. G. Rightor et al., J. Phys. Chem. B 101 (1997) 1950.

[4] A. P. Hitchcock et al., Microscopy \& Microanalysis, 11 (2005) this volume.

[5] Research funded by NWRI (Canada), AFMNet (Canada), NSERC (Canada), and the Canada Research Chair program. We thank D. Kilcoyne for development and maintenance of the 532 STXM. The Advanced Light Source is supported by the Director, Office of Energy Research, Office of Basic Energy Sciences, Materials Sciences Division of the U.S. Department of Energy under contract No. DE-AC03-76SF00098. 

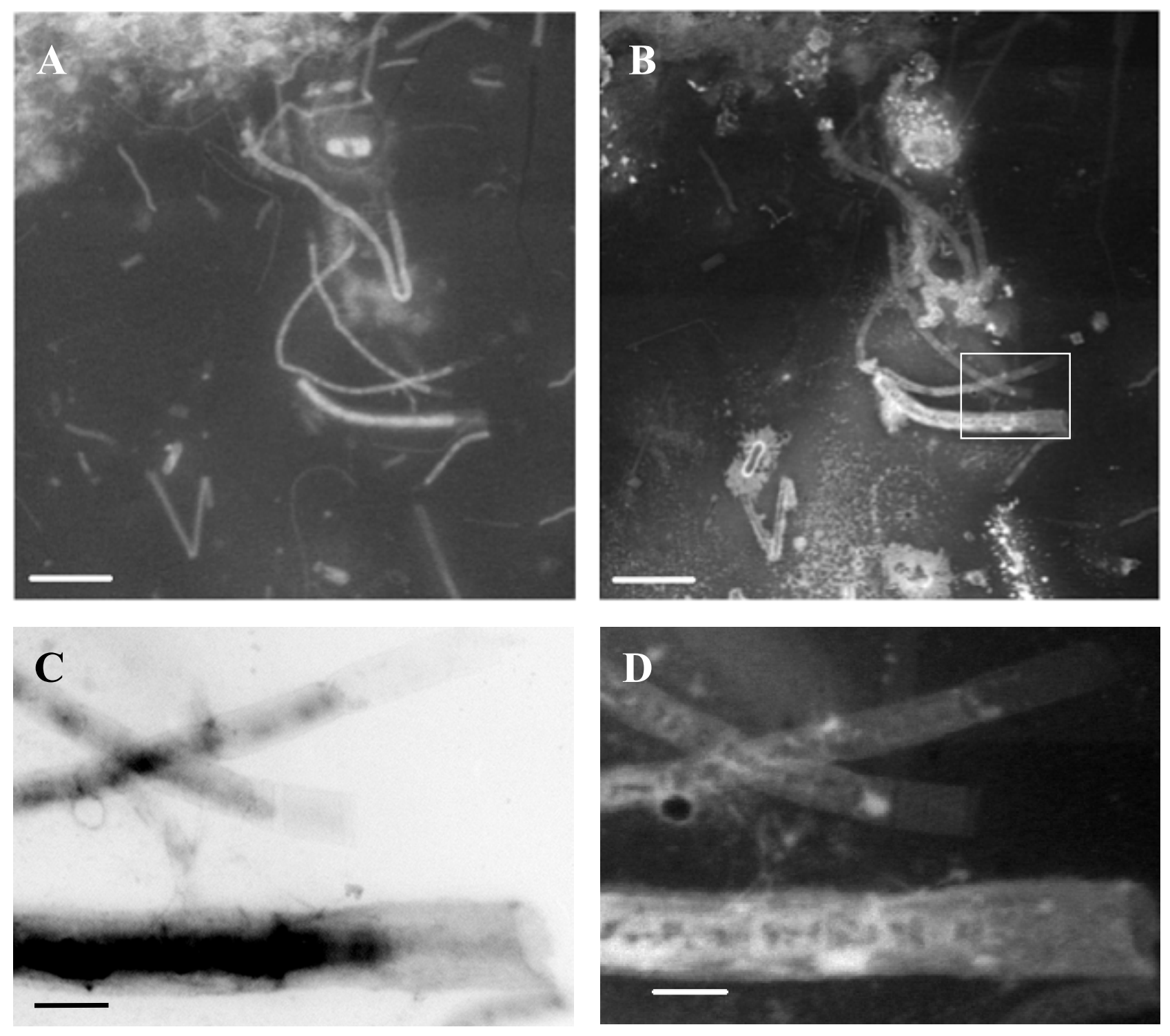

Fig. 1. Six week methanol-fed riverine biofilm that was frozen, cryo-sectioned and mounted on a Formvar-coated grid. A. STXM map of biological species (mostly protein as detected by absorption at $288.2 \mathrm{eV}$ minus that at $282 \mathrm{eV}$ ). B. STXM map of calcium (absorption at $352.5 \mathrm{eV}$ minus that at $350.3 \mathrm{eV}$ ) in the same area of biofilm. C. and D. Comparative imaging of the biofilm area delineated by a rectangle in B using TEM (C) and STXM Ca mapping (D). Scale bars $=5 \mu \mathrm{m}$ for $\mathrm{A}$ and $\mathrm{B}$. Scale bars $=1 \mu \mathrm{m}$ for $\mathrm{C}$ and $\mathrm{D}$. 